Supplement of Biogeosciences, 17, 2381-2396, 2020

https://doi.org/10.5194/bg-17-2381-2020-supplement

(C) Author(s) 2020. This work is distributed under

the Creative Commons Attribution 4.0 License.

(c) (1)

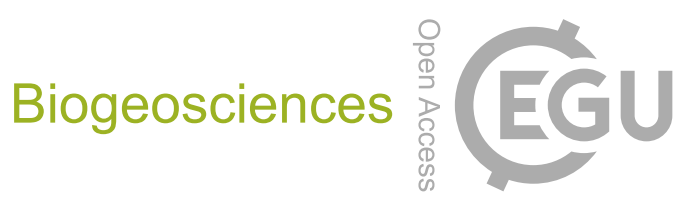

Supplement of

\title{
Evidence of changes in sedimentation rate and sediment fabric in a low- oxygen setting: Santa Monica Basin, CA
}

Nathaniel Kemnitz et al.

Correspondence to: Nathaniel Kemnitz (kemnitz@usc.edu)

The copyright of individual parts of the supplement might differ from the CC BY 4.0 License. 


\section{Supplement}

\section{${ }^{210} \mathrm{~Pb}$ Calibration and ${ }^{226} \mathrm{Ra}$}

5 To verify the ${ }^{210} \mathrm{~Pb}$ results for SMB sediments by gamma spectroscopy, several samples from MUC 9 were also analyzed by isotope dilution alpha spectroscopy following procedures described by Huh et al. (1987). Briefly, 0.5 grams of sediment was placed in Teflon beakers and spiked with a known amount of ${ }^{209}$ Po spike (USC \#65D). The ${ }^{209}$ Po spike was calibrated against a NIST certified ${ }^{210} \mathrm{~Pb}$ solution (NIST \#4337). The sediments, along with the ${ }^{209} \mathrm{Po}$, were subject to a series of acid digestions with $\mathrm{HCl}, \mathrm{HNO}_{3}, \mathrm{HF}$, and $\mathrm{HClO}_{4}$ or $\mathrm{H}_{2} \mathrm{O}_{2}$. After organics and silicates were dissolved, the solution was brought up in $50 \mathrm{~mL}$

$101 \mathrm{~N} \mathrm{HCl}$, and ascorbic acid was added to complex iron. A 12-mm silver disk was placed on the bottom of the Teflon beaker, and the solution was stirred and heated at $90{ }^{\circ} \mathrm{C}$ for 3-5 hours to plate polonium. After 3-5 hours of plating, the silver disk was removed from the solution, washed with DIW, and dried with acetone. The silver disk was then measured using alpha spectrometry. The plated disks were placed $10 \mathrm{~mm}$ from Surface Silicon Barrier Detectors (ORTEC, $300 \mathrm{~mm}$, average efficiency $=18 \%$ ) to measure the polonium isotopes. Backgrounds were counted regularly on each detector to monitor daughter

15 product build-up. A background count was subtracted from each sample, and equation S-1 was used to calculate the ${ }^{210} \mathrm{~Pb}$ in the dissolved sample.

$A_{210_{P b}}=\frac{210 N}{209 N} e^{\left(\lambda^{210} P o \Delta t\right)} A_{209 P o}$

20 where ${ }^{210} \mathrm{~N}$ and ${ }^{209} \mathrm{~N}$ are the background-corrected net counts of ${ }^{210} \mathrm{Po}$ and ${ }^{209} \mathrm{Po} ; \Delta \mathrm{t}$ is the time that elapsed from midpoint of plating and midpoint of counting; $\mathrm{A}_{209 \mathrm{Po}}$ is the activity of ${ }^{209} \mathrm{Po}$ spike added in dpm; and $\lambda$ is the decay constant for ${ }^{210} \mathrm{Po}$. We assumed that ${ }^{210} \mathrm{~Pb}$ and ${ }^{210} \mathrm{Po}\left(\mathrm{t}_{1 / 2}=138 \mathrm{~d}\right)$ are in equilibrium for SMB sediments. A comparison of these two counting methodologies is shown in Figure S-1 and provides convincing evidence that gamma and alpha spectroscopy yield identical results. 


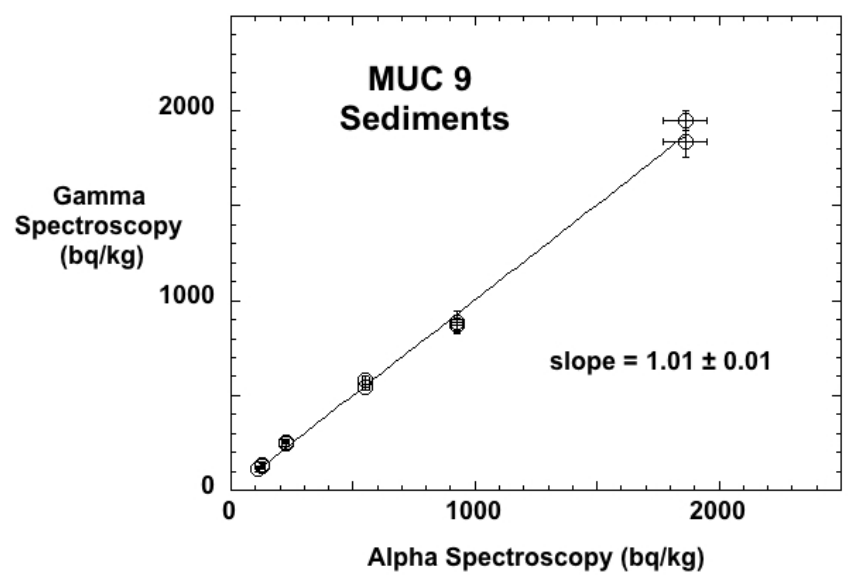

Figure S-1: Specific activities of ${ }^{210} \mathrm{~Pb}$ determined from gamma and alpha spectroscopy for Santa Monica Basin sediments (MUC-

9). The linear regression is forced through zero. All activities are corrected for salt content.

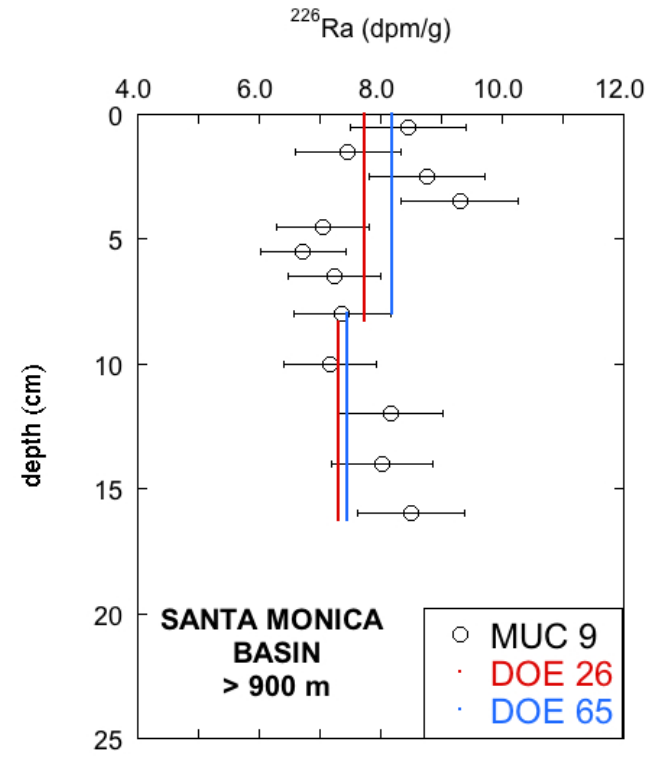

Figure S-2: ${ }^{226}$ Ra values for SMB cores MUC 9, DOE 65, and DOE 25. All three cores are sampled greater than 900 meters depth and within close proximity. MUC 9 was collected in 2016, and the measurement was based on gamma spectroscopy corrected for $10 \% \mathrm{Rn}$ loss. The other two cores were collected in the late 1980's and a composite sample was analysed by Rn ingrowth from the dissolved sediment (Christensen et al., 1993). 


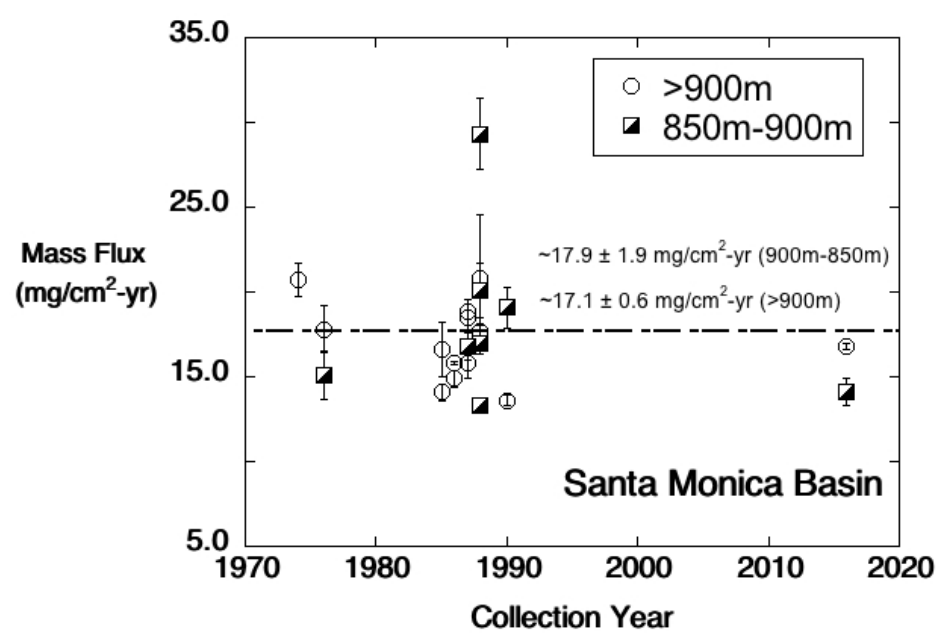

Figure S-3: Mass Flux vs. Collection Year for all cores greater than 850 meters in the SMB. Open circles and black and white squares are cores collected from $>900$ meters and $850-900$ meters depth regimes.

\section{Changes in LA Basin Land Use}

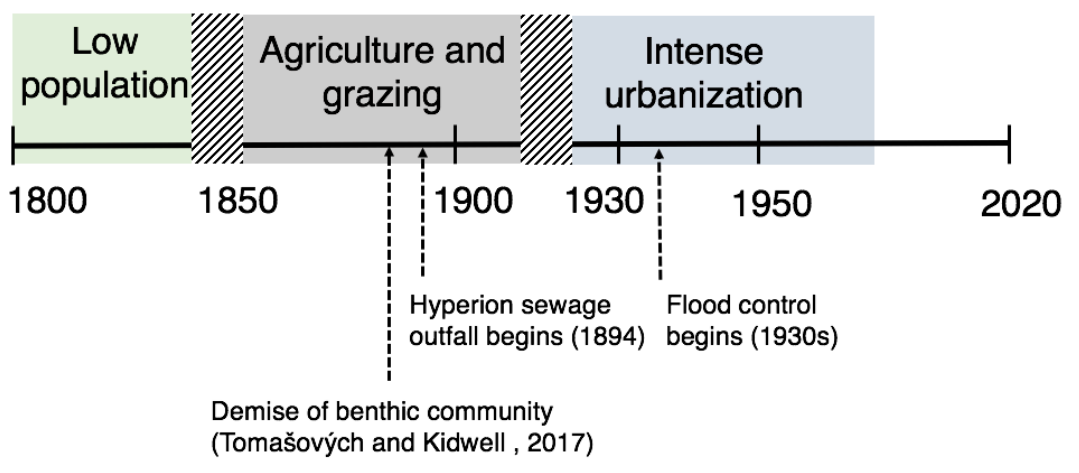

Figure S-4: Timeline of Los Angeles basin land usage beginning in 1800 until the present (2020). Note the demise of a shelly benthic community living on the Santa Monica Basin shelf (late 1800: Tomašových and Kidwell, 2017). 